Crecimiento de población y urbanización en Brasil en los años ochenta

\author{
Paulo de Martino Jannuzzi*
}

La población brasileña ha pasado por cambios demográficos significativos en las últimas décadas, de los cuales no se han enterado los encargados de la planeación pública. Este artículo discute dichos cambios -reducción del volumen anual de nacimientos, envejecimiento de población, disminución del tamaño de las familias, reducción del crecimiento poblacional de las grandes ciudades y expansión de las ciudades medianas del interior del país- y sus efectos sobre la demanda de bienes y servicios públicos.

Palabras clave: Brasil, cambio demográfico, urbanización, políticas públicas.

\title{
Introducción
}

En los últimos treinta años la población brasileña ha experimentado cambios significativos en su perfil demográfico. Los datos del censo demográfico de 1970 ya indicaban hace más de veinte años que el ritmo de crecimiento de población en Brasil tendía a disminuir y los resultados del censo de 1980 lo corroboraron. Aunque no se disponga de datos más completos del censo de 1991, es posible constatar una disminución aún más acentuada de las tasas de crecimiento poblacional para la década pasada.

Así, la población brasileña presenta una configuración poblacional muy distinta a aquella con la cual acostumbraban trabajar los órganos responsables de formular políticas públicas. Al escenario demográfico brasileño de los años noventa ya no pertenecen el crecimiento superior a $3 \%$ anual, la proporción de niños y jóvenes duplicándose cada 25 años o el crecimiento explosivo de São Paulo, Río de Janeiro y otras capitales.

Este estudio pretende discutir los principales cambios demográficos que ha experimentado la población brasileña en las últimas décadas -sobre todo en los años ochenta-, describir sus características demográficas actuales e indicar posibles tendencias futuras. A manera de conclusión se analizan brevemente las repercusiones de estos cambios en la demanda de bienes y servicios públicos.

* Analista de proyectos de la Dirección Adjunta de Análisis Socioeconómico de la Fundación SEADE en São Paulo, Brasil; maestría en Administración Pública en la SEADE/FGV y doctorado en Demografía en el IFCH/NEPO/Unicamp. 
Un cambio en el volumen anual de nacimientos

Uno de los más importantes cambios demográficos recientes es la disminución del volumen anual de nacimientos. Por primera vez en 50 años comenzaron a disminuir los nacimientos, reduciendo la tasa de crecimiento absoluto de la población.

En términos relativos, la reducción del ritmo de crecimiento poblacional se remonta a los años sesenta, cuando aquélla creció a tasas geométricas anuales de $2.88 \%$, contra $3.05 \%$ de la década anterior, que era la mayor tasa de crecimiento registrada en el país en el siglo xx. Esa alta tasa de los años cincuenta resultó de la diferencia progresiva entre las tasas de natalidad y mortalidad desde finales del siglo pasado. La natalidad permaneció relativamente estable hasta finales de los años cincuenta y la mortalidad comenzó a disminuir de manera acentuada a partir de los años cuarenta, como consecuencia de inversiones en higiene básica y salud pública, mejor control de enfermedades infecciosas e introducción en el país de medicamentos recién desarrollados (como antibióticos y sulfas) (Patarra, 1978; Martine y Camargo, 1984). Así, de 1940 a 1960 la mortalidad cayó cerca de $55 \%$, pasando de 20.6 por 1000 muertes a 9.6 por 1 000. Durante el mismo periodo la mortalidad infantil pasó de 164 por 1000 a 105 por 1000 .

A partir de los años sesenta, la entonces estable natalidad comenzó a sentir los cambios en el comportamiento reproductivo de las familias brasileñas, sobre todo en la segunda mitad de la década. La Tasa Global de Fecundidad (TGF) disminuyó de poco más de seis hijos por mujer entre 1940 y 1960 a 4.35 en 1980, como reflejo de una mayor escolarización, urbanización, participación femenina en el mercado de trabajo, divulgación del uso de anticonceptivos, difusión de nuevos patrones familiares y de consumo e incremento en la práctica de abortos inducidos (Merrick y Berquó, 1983). Se estima que durante la última década la TGF cayó aún más, llegando a 2.5 hijos por mujer en 1991 (Berquó, 1994).

El resultado práctico de esta disminución acentuada de la fecundidad durante la década pasada fue la reducción del número de nacimientos. Nótese que entre 1960 y 1980, aun con la disminución de la fecundidad, el ritmo de incremento anual de la población seguía en aumento, pues nuevas mujeres se incorporaban a la población en edad reproductiva (de 15 a 49 años). En la década de 1980 este grupo siguió creciendo, pasando de 30 millones en 1980 a 38 millones en 1991. Sin embargo, la disminución de la fecundidad fue tan intensa que incluso un número mayor de mujeres en edad reproductiva no fue suficiente para generar la cantidad anterior de nacimientos existentes en el pasado. Así, mientras que 
el saldo vegetativo (nacimientos menos muertes) anual promedio de los años setenta fue de 2596000 , el del siguiente periodo fue de 2536000 , rompiendo la tendencia histórica de aumentos sucesivos desde los años cuarenta, como se observa en el cuadro 1.

CUADRO 1

Crecimiento poblacional en Brasil, de 1940 a 2000

\begin{tabular}{lccc}
\hline & $\begin{array}{c}\text { Población } \\
\text { inicial } \\
\text { del } \\
\text { periodo }\end{array}$ & $\begin{array}{c}\text { Tasa de } \\
\text { crecimiento } \\
\text { geométrico } \\
\text { anual }\end{array}$ & $\begin{array}{c}\text { Saldo } \\
\text { vegatetivo } \\
\text { medio } \\
\text { anual }\end{array}$ \\
\hline $1940-1950$ & 41236000 & $2.33 \%$ & 1070000 \\
$1950-1960$ & 51944000 & $3.05 \%$ & 1818000 \\
$1960-1970$ & 70119000 & $2.88 \%$ & 2302000 \\
$1970-1980$ & 93139000 & $2.49 \%$ & 2596000 \\
$1980-1991$ & 119099000 & $1.93 \%$ & 2536000 \\
$1991-2000$ & 147054000 & $1.60 \%$ & 2350000
\end{tabular}

Fuente: Patarra, 1978; Carvalho, 1994 y censos demográficos.

Ha de observarse que si la esperanza de vida al nacer no hubiera aumentado de 60 a 66 años en el periodo de 1980 a 1989 (Médici, 1991), los incrementos vegetativos serían aún menores. En los años noventa, de acuerdo con las proyecciones de crecimiento poblacional de Carvalho (1994), los incrementos vegetativos serían de 2350000 personas.

Una reducción tal en el ritmo de crecimiento demográfico hará que la población brasileña no alcance en el año 2000 los 212 millones previstos en los años setenta, sino $20 \%$ menos $(169 \mathrm{mi}-$ llones). Además, a largo plazo, esta disminución del ritmo llevará a la población a converger en un estado estacionario, con distribución por edades constante y crecimiento nulo. Sin embargo, todavía falta mucho tiempo para eso. Con base en la ausencia de flujos migratorios internacionales y en ciertas suposiciones bastante plausibles del comportamiento de la fecundidad y mortalidad durante las próximas décadas, Carvalho (1993) sugiere que la población brasileña se estacionará por debajo de los 250 millones, con tasas de crecimiento insignificantes a partir del año 2060 (gráfica 1). 


\section{GRÁFICA 1}

Evolución poblacional brasileña entre 1890 y 2100

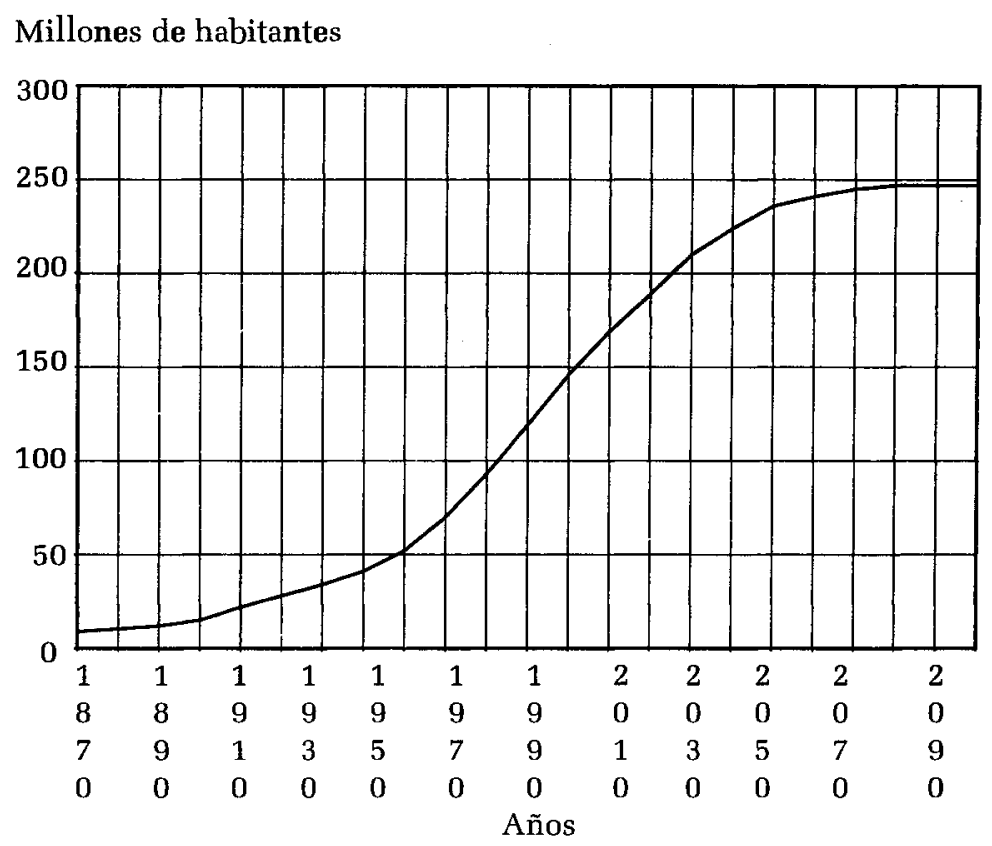

Fuente: censos demográficos; Carvalho, 1993 y 1994.

\section{El cambio en la distribución por edades}

La idea de que Brasil es un país de niños y jóvenes forma parte del sentido común del brasileño y, a diferencia de otras creencias, es una realidad. Aunque sólo por ahora, pues la población está envejeciendo.

Desde el censo de 1980 la distribución por edades de la población brasileña comenzó a reflejar los efectos de la disminución monotónica de la fecundidad iniciada en la segunda mitad de la década de los años sesenta (Berkovich y Vellozo, 1984). En 1970 los menores de 15 años constituían $43 \%$ de la población, en 1991 pasaron a representar $35 \%$. Por el contrario, el peso relativo de las personas de 65 años o mayores pasó de 3 a $5 \%$ en el mismo perio- 
do (cuadro 2). Como ilustran las gráficas 2 y 3 , la pirámide de edades de 1970 es típica de una situación de alta natalidad, mientras que la de 1991 ya representa un estrechamiento relativo de la base, característico de niveles más bajos de natalidad.

CUADRO 2

Distribución por edades de la población brasileña: 1970-2060 (porcentaje en relación a la poblacion total de cada año)

\begin{tabular}{|c|c|c|c|c|c|}
\hline Grupos de edad & 1970 & 1980 & 1991 & 2000 & 2060 \\
\hline $0-14$ & 42.6 & 38.8 & 35.5 & 29.7 & 20.6 \\
\hline $15-64$ & 54.3 & 57.2 & 59.7 & 65.2 & 64.7 \\
\hline $65+$ & 3.1 & 4.0 & 4.8 & 5.1 & 14.7 \\
\hline Total & 100.0 & 100.0 & 100.0 & 100.0 & 100.0 \\
\hline Población total* & 93139 & 119099 & 147054 & 169471 & 244926 \\
\hline
\end{tabular}

* En miles.

Fuente: resultados preliminares del censo de 1991 y Carvalho (1994).

\section{GRÁFICA 2}

Pirámide de edades; Brasil, 1970

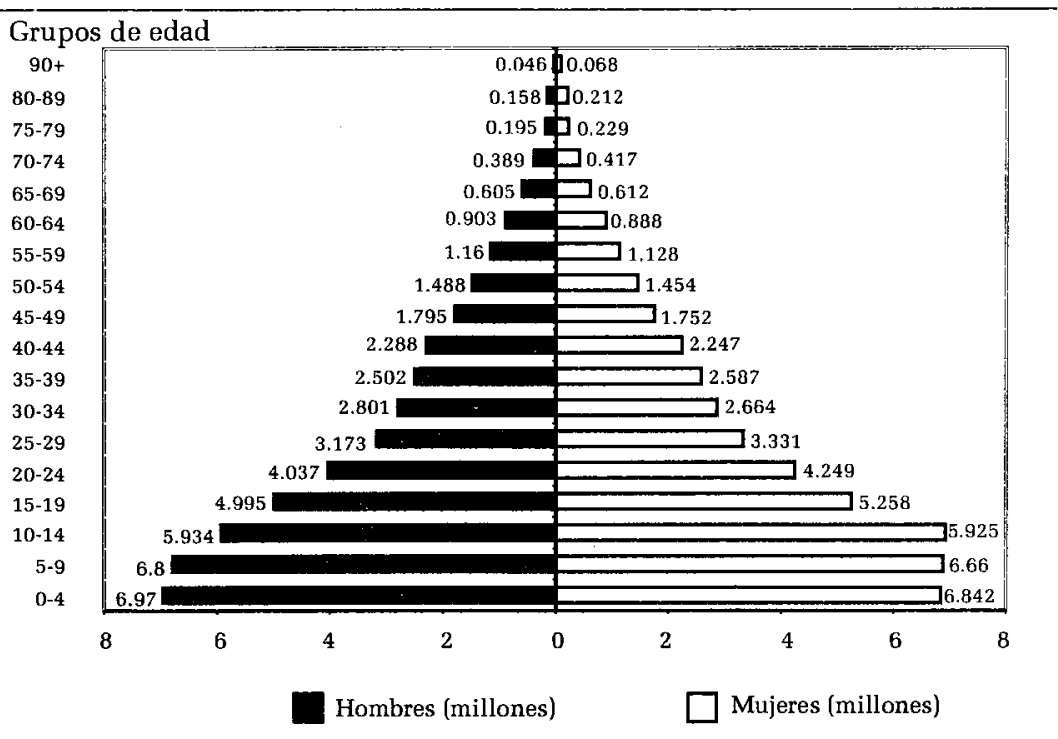

Fuente: resultados preliminares del censo de 1991. 
GRÁFICA 3

Pirámide de edades; Brasil, 1991

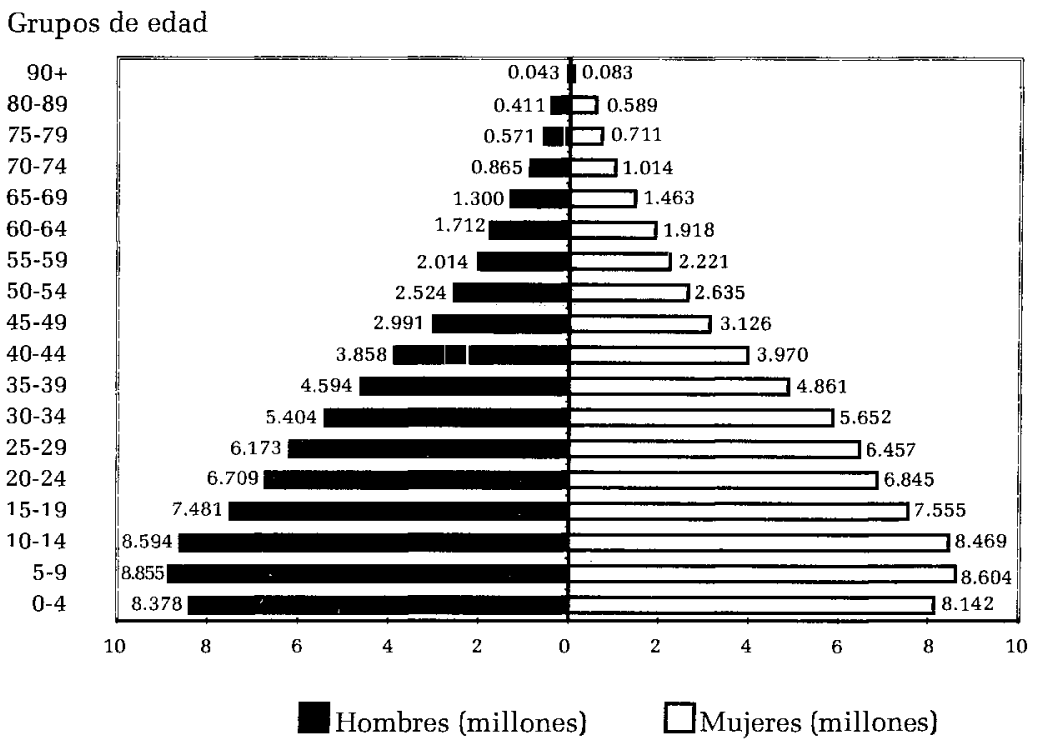

Fuente: resultados preliminares del censo de 1991.

En el caso hipotético de que la población brasileña llegue a un estado estacionario para el año 2060 (Carvalho, 1993), la estructura por edades tomaría una forma completamente distinta. Los niños y adolescentes menores de catorce años pasarían a representar poco más de $20 \%$ de la población y el conjunto de personas mayores de 65 años hasta $15 \%$. La pirámide de edades se convertiría en rectángulo.

Es importante señalar que la distribución por edades de la población tomada como conjunto único encubre diferencias significativas en términos de los diversos subgrupos poblacionales, estratificados por región o por ingreso. Véanse, por ejemplo, las diferencias entre las pirámides de edad en 1991 de São Paulo y Amazonas (gráficas 4 y 5), que ilustran distintos regímenes demográficos. 
GRÁFICA 4

Pirámide de edades; São Paulo, 1991

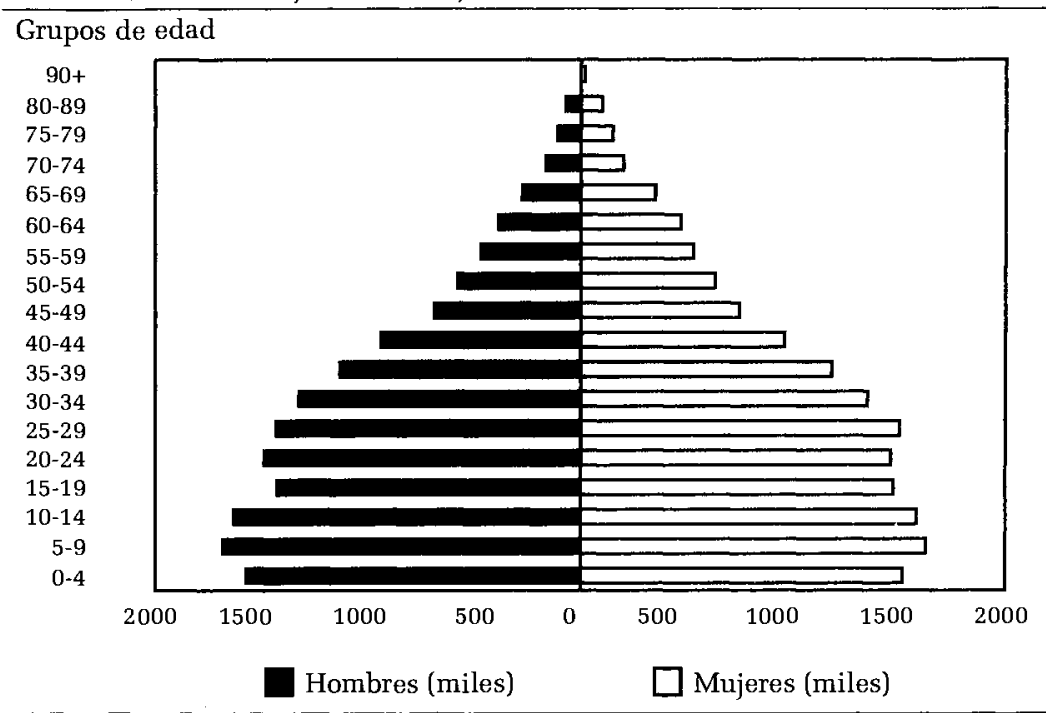

Fuente: resultados preliminares del censo de 1991.

GRÁFICA 5

Pirámide de edades; Amazonas, 1991

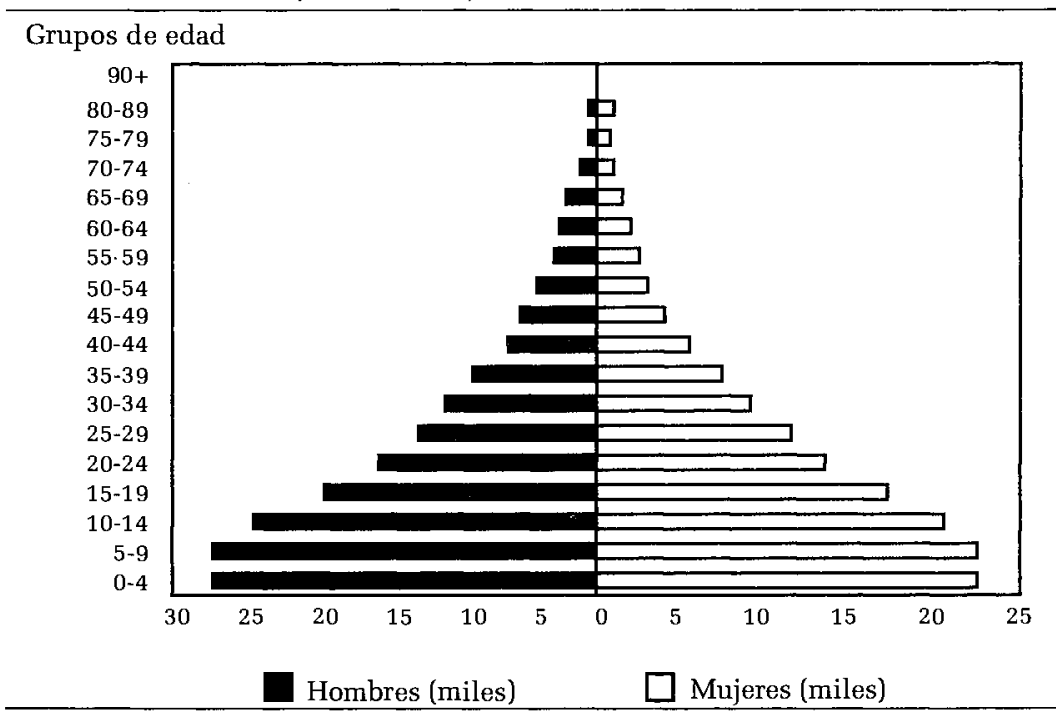

Fuente: resultados preliminares del censo de 1991. 
En cuanto a las diferencias en la distribución por edades en cada estrato socioeconómico, aún no hay suficientes datos para presentar resultados. Dentro de todo, es interesante referirnos a los resultados presentados por Camarano y Beltrão (1990), cruzando la información de distribución por edades con la de ingreso familiar mensual medio para 1980. Como se observa en el cuadro 3, las distribuciones por edad de las poblaciones de bajo y alto ingresos son muy distintas a las observadas en las familias de ingresos más altos. La participación porcentual de niños y adolescentes en el conjunto es menor en los estratos de ingresos familiares más altos. Tales diferencias resultan principalmente de los diferenciales de fecundidad de las mujeres, que van desde seis hijos por mujer en los estratos más pobres a dos hijos por mujer en el otro extremo (estimaciones para el periodo 1975-1980). Los distintos niveles de mortalidad que se tienen según ingresos para los distintos estratos también contribuyen a las diferencias de las estructuras por edad observadas, aunque en menor grado.

\section{CUADRO 3}

Estructura por edad de los estratos de población en porcentaje según el nivel de ingreso medio mensual familiar para 1980 (en salarios mínimos)

\begin{tabular}{rrrrrrrr}
\hline $\begin{array}{l}\text { Grupos } \\
\text { de } \\
\text { edad }\end{array}$ & \multicolumn{4}{c}{ Nivel de ingreso familiar (en salarios minimos) } & $\begin{array}{c}\text { Promedio } \\
\text { en }\end{array}$ \\
\cline { 2 - 7 } & $0-1$ & $1-3$ & $3-5$ & $5-10$ & +10 & Brasil \\
\hline $0-4$ & 18 & 16 & 12 & 10 & 9 & 14 \\
$5-14$ & 27 & 27 & 25 & 21 & 17 & 24 \\
$15-64$ & 48 & 53 & 60 & 66 & 70 & 58 \\
$65+$ & 7 & 4 & 3 & 3 & 4 & 4 \\
+ & & & 100 & 100 & 100 & 100 & 100 \\
Total & 100 & 100 & & & & \\
\hline
\end{tabular}

Fuente: Camarano y Beltrão, 1990: 11-14.

A juzgar por los niveles actuales de fecundidad, los patrones reproductivos por estrato socioeconómico no difieren. Berquó (1994:16) sugiere que esto es resultado de la alta prevalencia del uso de anticonceptivos en la población femenina (en 1986, 70\% de las mujeres en edad reproductiva, casadas o en unión consensual utilizaron métodos anticonceptivos). No obstante la inercia demográfica, las diferencias en las estructuras por edad según es- 
trato socioeconómico deben haber sufrido alteraciones significativas en la última década.

\section{Cambios en la composición familiar}

El tamaño medio de la familia brasileña está disminuyendo, como corolario de la desaceleración del crecimiento poblacional. En 1950 la mitad de las familias brasileñas estaba compuesta por cinco o más miembros. Patarra et al. (1991) estimaron que en 1984 las familias de este tamaño representaban poco más de un tercio solamente. Así, en 1987 el tamaño medio de la familia brasileña era de cuatro miembros (Souto-Maior, 1990). A juzgar por el número medio de personas por domicilio, registrado en el censo de 1991, la familia media nacional ya posee menos de cuatro miembros. Aún hay diferencias significativas entre regiones y niveles socioeconómicos, aunque cada vez menores.

El tamaño medio de la familia también es consecuencia de cambios en los patrones de arreglo familiar, que resultan de cambios socioculturales y socioeconómicos como aumento de la edad al casarse, de la tasa de divorcios y separaciones, ingreso cada vez mayor de las mujeres a la fuerza de trabajo, etc. En este sentido, Berquó et al. (1990) señalan, para los últimos veinte años, un incremento de personas que viven solas, de familias monoparentales (encabezadas principalmente por mujeres) y de parejas sin hijos, así como la disminución porcentual de familias extendidas (con parientes y agregados).

CUADRO 4

Tamaño medio de la familia brasileña entre 1970 y 1987

\begin{tabular}{lccccc}
\hline Región & 1970 & 1980 & 1983 & 1985 & 1987 \\
\hline Norte & 5.5 & 5.1 & 4.7 & 4.6 & 4.5 \\
Noreste & 5.1 & 4.8 & 4.7 & 4.6 & 4.5 \\
Sureste & 4.6 & 4.1 & 4.0 & 3.8 & 3.8 \\
Sur & 4.9 & 4.2 & 4.1 & 3.9 & 3.8 \\
Centro-oeste & 5.1 & 4.6 & 4.4 & 4.2 & 4.1 \\
Brasil & 4.8 & 4.2 & 4.2 & 4.1 & 4.0 \\
\hline
\end{tabular}

Fuente: Souto-Maior, 1990. 
El nuevo patrón de urbanización y distribución espacial de la población

En la literatura sobre el proceso de urbanización en Brasil, sea cual fuere el criterio empleado para "urbano" -aquel adoptado oficialmente por el ${ }_{\mathrm{IBGE}},{ }^{1}$ o el de "cualquier poblado con más de 2000 habitantes" (Katzman, 1986), u otro más restrictivo, como "la población (urbana y rural) que vive en municipios con más de 20000 habitantes" (Martine et al., 1988)-, hay un cierto consenso de que no se puede negar el ritmo acelerado de la transición rural-urbana de la población brasileña. En cuarenta años, de 1940 a 1980, la población residente en sitios con más de 2000 habitantes creció de 25 a $65 \%$ de la población total del país; la mitad del tiempo que tomó el proceso equivalente en Estados Unidos, país que tampoco se puede clasificar como un ejemplo histórico de urbanización lenta y gradual.

Consolidando las tendencias de las décadas pasadas, el censo de 1991 revela un país en el cual más de 80\% de su población vive en ciudades mayores a 20000 habitantes, y cerca de $75 \%$ en áreas consideradas como urbanas.

CUADRO 5

Urbanización en Brasil entre 1940 y 1991

\begin{tabular}{lcc}
\hline Año & $\begin{array}{c}\text { Porcentaje de } \\
\text { criterio } \\
\text { oficial }\end{array}$ & $\begin{array}{c}\text { Porcentcie de población viviendo en } \\
\text { municipios con más de } \\
\text { 20 ooo habitantes }\end{array}$ \\
\hline 1940 & 31.2 & 16.0 \\
1950 & 36.2 & 21.1 \\
1960 & 44.7 & 28.8 \\
1970 & 55.9 & 41.1 \\
1980 & 67.6 & 53.6 \\
1991 & 75.0 & 80.5 \\
\hline
\end{tabular}

Fuente: resultados preliminares del censo de 1991; Martine et al. (1988) y Martine (1992).

1 De acuerdo con el criterio oficial, la población urbana es aquella que vive dentro de los límites geográficos de la sede del municipio o distrito, tal y como están definidos en la ley municipal. 
Históricamente, la urbanización fue un fenómeno difundido por el territorio, relativamente independiente del desarrollo de la industria local, y se llevó a cabo con distintos ritmos según la localidad y el tamaño de las ciudades (Patarra, 1978; Faria, 1984). Así, entre 1940 y 1980 la región del centro-oeste presentó las tasas de crecimiento urbano más altas del país, como consecuencia de la construcción de Brasilia y la ocupación de la frontera agrícola. En este mismo periodo el noreste presentó las tasas más bajas (Martine, 1988). En términos de tamaño de ciudad, durante el mismo periodo, la población urbana creció a tasas más altas en los municipios medianos, de entre 100000 mil y 500000 habitantes. Crecen a tasas menores los municipios grandes y las megalópolis ${ }^{2}$ llegan a reunir en 1980 a casi la tercera parte de la población brasileña. Fue lo que en los años setenta se llamó la megalopolización del sistema urbano brasileño.

Para la década pasada, Martine (1992) señaló una inflexión en la tendencia a la megalopolización de la población brasileña. Como consecuencia de la crisis de los años ochenta y de la interiorización del desarrollo económico, disminuyeron los movimientos migratorios hacia los grandes centros, dirigiéndose hacia ciudades más cercanas y medianas del interior del territorio nacional. Como constata el autor, "la progresiva concentración de la población en ciudades cada vez mayores, que parecía inexorable se revirtió" (Martine, 1992: 13).

La metropolización iniciada en los cuarenta en São Paulo y Río de Janeiro y extendida a otras capitales brasileñas en los años sesenta parece haberse agotado. De hecho, la tasa de crecimiento del conjunto de las zonas metropolitanas entre 1980 y 1991 permaneció, por primera vez desde 1950 , muy cercana a la media nacional. En el último decenio las zonas metropolitanas de São Paulo, Recife y Río de Janeiro presentaron las menores tasas de crecimiento demográfico. Las tasas de crecimiento de otras metrópolis, aunque superiores a la media brasileña, fueron significativamente menores a las que se observaron en los años setenta. Por el contrario, las localidades ubicadas sobre la frontera amazónica, los balnearios y varios municipios del interior paulista están entre las ciudades con mayor crecimiento demográfico. A este grupo pertenecen también varios municipios periféricos a las grandes ciudades. ${ }^{3}$

2 Megalópolis se refiere aquí a las aglomeraciones urbanas de gran volumen poblacional (más de tres millones de personas), como las zonas metropolitanas de São Paulo y Río de Janeiro.

3 Esta desaceleración del crecimiento de las regiones densamente pobladas y 
En la década pasada la parcela poblacional de los municipios grandes y muy grandes (con más de $\mathbf{5 0 0} 000$ habitantes) se redujo, pues pasó a representar $26 \%$ de la población, contra $32 \%$ de 1980. Por otro lado, como puede observarse en la gráfica 6 , que ilustra la distribución de la población en el sistema de ciudades, se verifica que los municipios de entre 20000 y 100000 habitantes pasaron en 1991 a reunir cerca de la tercera parte de la población brasileña, como resultado del crecimiento acentuado de los municipios que en 1980 tenían hasta 20000 habitantes.

\section{GRÁFICA 6}

Distribución de la población en el sistema urbano; Brasil, 1991

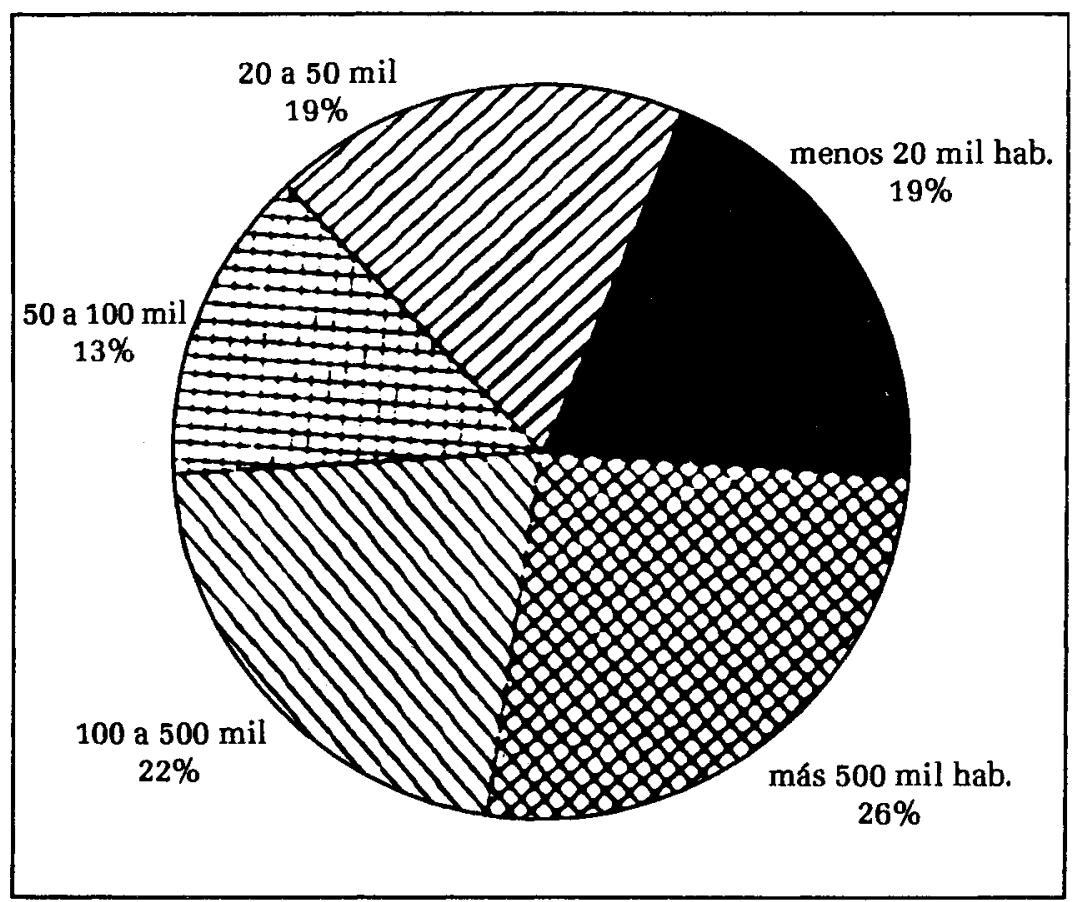

Fuente: resultados preliminares del censo de 1991.

el consecuente aumento demográfico de las ciudades menores ya la había notado Katzman desde los años sesenta. Sin embargo, en la década de los ochenta este fenómeno parece haberse generalizado. 
En cuanto a la distribución regional de la población, los primeros datos del censo revelan las mismas tendencias de las décadas pasadas (mapa 1). El norte y el centro-oeste siguen aumentando su participación en el conjunto poblacional del país, por la persistencia de tasas y fecundidad mayores, principalmente la del flujo migratorio. Ambas regiones crecieron a tasas superiores a $3 \%$ anual, duplicando sus participaciones relativas de 1950, en detrimento del sur y el noreste. En 1991, el norte y centro-oeste reunían, respectivamente, 7 y $6.4 \%$ de la población del país, correspondiendo al sur y noreste 15 y $29 \%$. La región del sureste sigue siendo la más poblada, con su histórico $43 \%$ de la población total (gráfica 7).

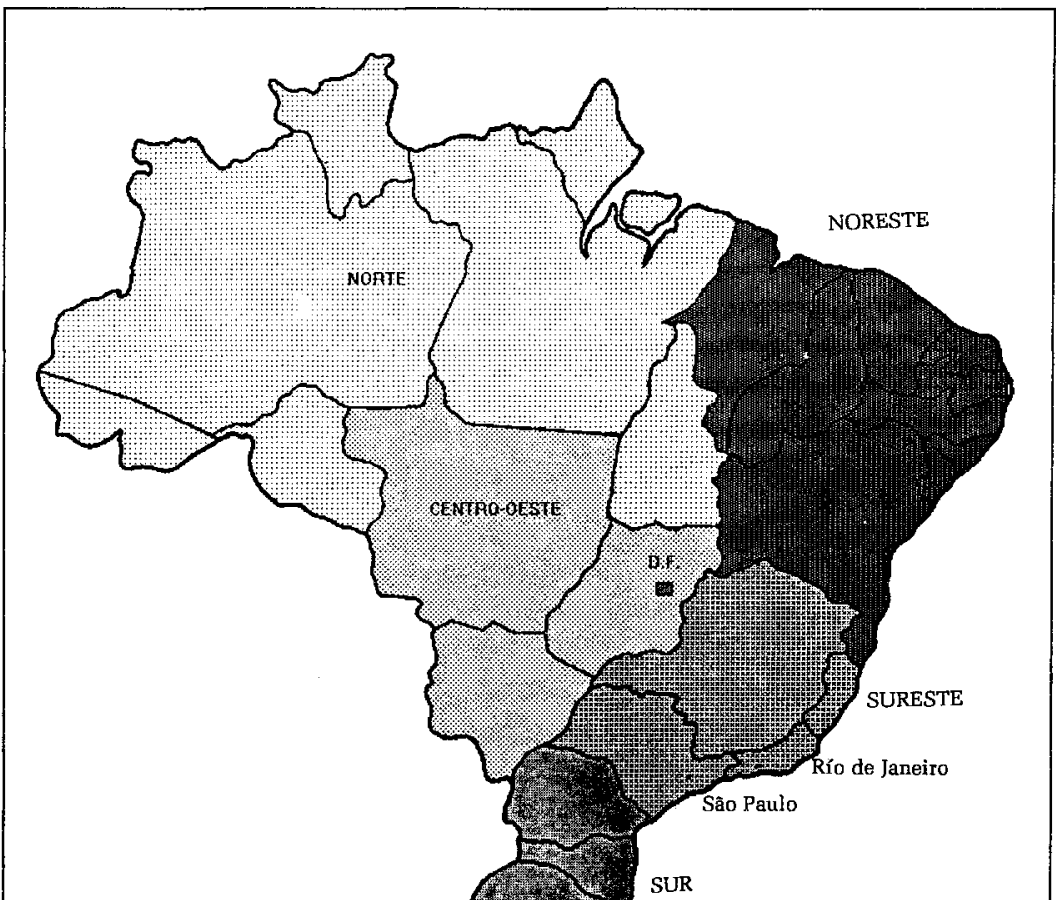




\section{GRÁFICA 7}

Distribución de la población por grandes regiones; Brasil, 1991 (en porcentajes)

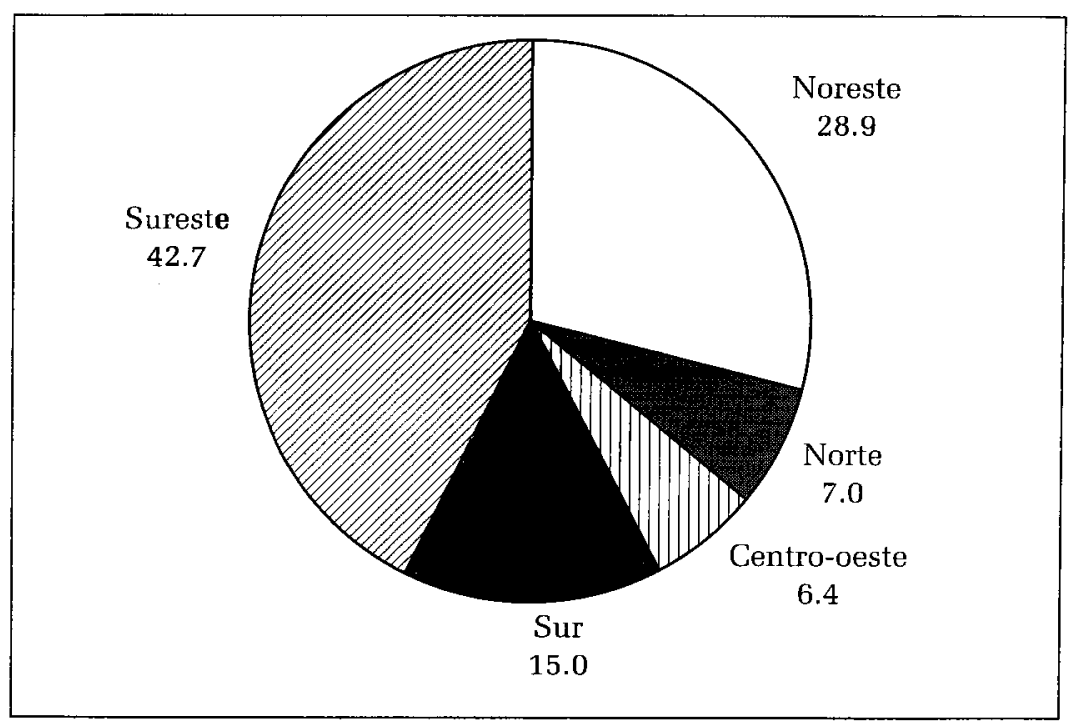

Fuente: resultados preliminares del censo de 1991.

Aunque en todas las regiones los pocos municipios grandes y muy grandes retengan a una parte sustancial de la población, hay algunas diferencias en la distribución poblacional para los sistemas urbanos regionales. En el sur y sureste hay cierto equilibrio en la distribución de la población entre las ciudades de distinto tamaño. En el norte y centro-oeste los municipios entre 20000 y 100000 habitantes tienen mayor importancia en el sistema urbano. En el noreste las ciudades pequeñas (hasta 50000 habitantes) reúnen $50 \%$ de la población.

Los cambios demográficos y las nuevas demandas de bienes y servicios

Los cambios demográficos señalados aquí -reducción del número de nacimientos y del tamaño medio de las familias, envejecimiento poblacional, urbanización intensa y disminución en el ritmo de crecimiento poblacional en los grandes municipios- tienen efectos sobre la formulación de políticas públicas en la medida en que la demanda de bienes y servicios adquiere una nueva configuración. 
CUADRO 6

Distribución de la población brasileña por grandes regiones y por tamaño de ciudad (expresada en miles de habitantes)

\begin{tabular}{lrrrrrr}
\hline $\begin{array}{l}\text { Tamaño } \\
\text { de las } \\
\text { ciudades } \\
\text { (miles) }\end{array}$ & Norte & Noreste & Sureste & \multicolumn{1}{c}{ Sur } & $\begin{array}{r}\text { Centro- } \\
\text { oeste }\end{array}$ & Total \\
\hline Hasta 2 & - & 18.3 & 40.7 & 17.5 & 15.0 & 91.4 \\
$2-10$ & 563.1 & 2779.5 & 3353.1 & 2347.4 & 1110.0 & 10152.8 \\
$10-20$ & 1328.6 & 7544.7 & 4859.8 & 3441.1 & 1259.2 & 18433.4 \\
$20-50$ & 2281.1 & 11842.5 & 7706.4 & 4437.0 & 1771.4 & 28038.3 \\
$50-100$ & 2196.3 & 6232.8 & 7172.2 & 3123.1 & 770.0 & 19494.4 \\
$100-250$ & 1086.1 & 3666.1 & 8339.4 & 4331.1 & 1050.1 & 18472.7 \\
$250-500$ & 552.6 & 2737.7 & 7988.8 & 1853.8 & 402.8 & $\mathbf{1 3 5 3 5 . 7}$ \\
$500-1000$ & - & 2528.8 & 4835.2 & - & 1448.3 & 8812.4 \\
$\quad+1000$ & 2256.2 & 5142.2 & 18444.8 & 2578.4 & 1601.1 & 30022.8 \\
Total & 10264.0 & 42492.6 & 62740.4 & 22129.4 & $\mathbf{9 4 2 7 . 9}$ & 147054.0 \\
\hline
\end{tabular}

Fuente: resultados preliminares del censo de 1991.

Varios autores han discutido las repercusiones de estos procesos en las políticas sociales (Martine y Camargo, 1984; Martine, 1992; Chadad, 1990 y Carvalho, 1994). De modo general, observan que la reducción del crecimiento de la población y la alteración de su distribución por edades tienden a alterar la demanda de servicios públicos -sobre todo de salud, educación y previsión social. Un menor número de nacimientos disminuye la demanda de servicios médicos de pediatría, ginecología y obstetricia; en la educación, implica una reducción en el número de ingresantes a guarderías y escuelas. Por otro lado, el envejecimiento de la población exige costosas inversiones para atender enfermedades geriátricas y degenerativas, y aumenta la demanda de servicios preventivos y de instalaciones públicas de esparcimiento para los ancianos.

La reducción del tamaño de la familia, sobre todo de bajos ingresos, podría significar mayor bienestar material y alimenticio per cápita. Las altas tasas de urbanización, aunque facilitan la universalización de los servicios de salud y educación, exigen inversiones cada vez mayores en infraestructura sanitaria, de transporte, habitacional y de esparcimiento. La disminución en el ritmo de crecimiento de los grandes centros urbanos, si se mantuviese como fenómeno no meramente de coyuntura, puede desahogar la necesidad de expansión acelerada de la oferta de servicios e 
instalaciones urbanos para las administraciones locales. En contraparte, exigirá un ritmo más intenso en las ciudades pequeñas y medianas, donde el presupuesto puede ser más escaso e insuficiente para tales inversiones.

Naturalmente no son sólo los cambios demográficos los que redefinen estas demandas sociales. El ceteris paribus macroeconómico implícito en el análisis anterior está lejos de ser una abstracción factible. Las transformaciones en el mercado de trabajo, los cambios socioeconómicos y las alteraciones en los estilos de vida y en los valores culturales también deben tomarse en cuenta para el diseño de las nuevas políticas públicas. Los datos del censo demográfico de 1991 que están por aparecer, y otras fuentes periódicas de información socioeconómica, podrían ayudar a develar esta nueva realidad.

\section{Bibliografía}

Bercovich, A. y H. Vellozo (1984), "Notas sobre aparentes contradições na estrutura por idade e sexo no Censo Demográfico de 1980", Revista Brasileira Estatística, vol. 46, núms. 181-182, pp. 53-121.

Berquó, Elza (1994), “Uma queda (cirurgica) na fecundidade”, publicación especial de la revista Imprensa, núm. 76, pp. 16-20.

- et al. (1990), "Arranjos familiares não-canõnicos no Brasil”, Anais do VII Encontro Nacional de Estudos Populacionais, São Paulo, ABEP, vol. 2, pp. 55-98.

Camarano, A.A. y K.I. Beltrão (1990), “Dinámica Demográfica por nivel de renda", Reporte Técnico 08/90, Río de Janeiro, ENCE/IBGE.

Carvalho, J.A.M. (1993), Crescimento Populacional e Estrutura Demográfica no Brasil, Belo Horizonte, CEDEPLAR/UFMG.

_ (1994), "Um Brasil mais velho e mais estavel”, publicación epecial de la revista Imprensa, núm. 76, pp. 4-11.

Chadad, J.P.C. (1990), "População, força de trabalho e desemprego no Brasil: 1960-1989", Anais do VII Encontro Nacional de Estudos Populacionais, São Paulo, ABEP, vol. 2, pp. 51-82.

Faria, Vilmar (1984), "Desenvolvimento, urbanização e mudanças na estrutura do emprego: a experiência brasileira dos últimos trinta anos", en B. Sorj et al. (comps.), Sociedade e Política no Brasil pós-64, São Paulo, Brasiliense, pp. 118-163.

Katzman, M. (1986), “Urbanização no Brasil a partir de 1945”, en E. Bacha y H. Klein (comps.), A Transição Incompleta: Brasil desde 1940, Río de Janeiro, Paz e Terra, pp. 177-223.

Martine, G. (1992), "Processos Recentes de Concentraçao e Desconcentraçao Urbana no Brasil: Determinantes e Implicações", Documento de Trabajo/11, Brasilia, Instituto sPN.

y L. Camargo (1984), "Crescimento e distribução da população 
brasileira: tendências recentes", Revista Brasileira de Estudos Populacionais, Campinas, vols. 1-2, núm. 1, pp. 99-144.

- et al. (1988), "A urbanização no Brasil: retrospectiva, componemtes e perspectivas", en Anais do VI Encontro Nacional de Estudos Populacionais, Olinda, ABEP, pp. 19-64.

Médici, A.C. (1991), "Sem Régua Nem Compasso", Reporte Técnico 06/91, Río de Janeiro, ENCE/IBGE.

Merrick, T. y E. Berquó (1983), The Determinants of. Brazil's Recent Rapid Decline in Fertility, Washington, National Academy of Sciences.

Patarra, Neide (1978), "Dinãmica Populacional e Urbanização no Brasil: o período pós-30", en B. Fausto (comp.), História Geral da Civilização Brasileira, São Paulo, Difel, t. 3, v. 4, pp. 249-268.

et al. (1991), "Demografia", Documento de Trabajo QS/11, São Paulo, FUNDAP.

Souto-Maior, H.P. (1990), “Familia no Nordeste: 1970-1987”, en Anais do VII Encontro Nacional de Estudos Populacionais, São Paulo, ABEP, vol. 1, pp. 161-184. 
